Research Article

\title{
Frequency Response Function-Based Finite Element Model Updating Using Extreme Learning Machine Model
}

\author{
Yu Zhao 1 and Zhenrui Peng (iD \\ School of Mechanical Engineering, Lanzhou Jiaotong University, Lanzhou 730070, China \\ Correspondence should be addressed to Zhenrui Peng; pengzr@mail.lzjtu.cn
}

Received 27 February 2020; Revised 3 August 2020; Accepted 11 September 2020; Published 5 October 2020

Academic Editor: Francesco Franco

Copyright (c $2020 \mathrm{Yu}$ Zhao and Zhenrui Peng. This is an open access article distributed under the Creative Commons Attribution License, which permits unrestricted use, distribution, and reproduction in any medium, provided the original work is properly cited.

\begin{abstract}
A frequency response function- (FRF-) based surrogate model for finite element model updating (FEMU) is presented in this paper. Extreme learning machine (ELM) is introduced as the surrogate model of the finite element model (FEM) to construct the relationship between updating parameters and structural responses. To further improve the generalization ability, the input weights and biases of ELM are optimized by Lévy flight trajectory-based whale optimization algorithm (LWOA). Then, LWOA is also applied to obtain the best updating results, where the objective function is defined by the difference between analytical FRF data and experimental data. Finally, a plane truss is used to demonstrate the performance of the proposed method. The results show that, compared with second-order response surface (RS), radial basis function (RBF), traditional ELM, and other optimized ELM, a LWOA-ELM model has higher prediction accuracy. After updating, the FRF data and frequencies have a significant match to the experimental model. The proposed FEMU method is feasible.
\end{abstract}

\section{Introduction}

Due to the capacity for structural identification and health monitoring, finite element method has attracted much more attentions in the past few years. However, an initial finite element model (FEM) cannot reflect the actual structure precisely, because of simplifications and idealizations (ideal boundary conditions, material properties, etc.) while constructing the FEM [1-3]. In order to be used for further analysis, the initial FEM should be updated to minimize the error between the analytical responses and the experimental ones, which is called finite element model updating (FEMU) [4]. Several methods for FEMU have been developed. Among these methods, vibration-based techniques are widely used. This kind of technique can be classified into two main categories: the methods based on modal data and the ones based on frequency response function (FRF) data [5-9]. For the former, natural frequencies and mode shapes are introduced to define the objective function [10]. For the latter, FRF data provide enough response information directly without modal analysis, which can increase the accuracy of model updating. The FRF-based methods have been gradually applied in FEMU $[11,12]$.

In addition, the model updating requires dozens of iterations to compute the analytical responses, which is timeconsuming. The surrogate models, such as response surface (RS), Kriging model, radial basis function (RBF), neural network (NN), and support vector machine (SVM), are widely used to construct the relationship between the input parameters and output responses to replace the original FEM. Marwala [13] introduced multilayer perceptron (a neural network) as the surrogate model to update an H-type structure. Ren and Chen [14] presented a quadratic polynomial response surface-based method. A simply supported beam and a concrete bridge demonstrated the applicability and efficiency of the proposed method. Wang et al. [15] applied the Kriging model in a honeycomb Sandwich beam to reduce the solving time. Zhang and Hou [16] presented a SVM based response surface model for vehicle chassis components. With good generalization performance and fast learning speed, a learning method called extreme learning machine (ELM) for single-hidden layer feedforward 
neural networks (SLFNs) has received many applications in data analysis, protein sequence classification, feature selection and clustering learning, etc. [17-19]. Although ELM does not need to adjust so many parameters during training process, generalization performance of ELM could be affected by the input weights and the biases. To improve the performance, several methods, such as genetic algorithm (GA) and particle swarm optimization (PSO), have been proposed to optimize the input weights and biases of ELM $[20,21]$.

Besides computational cost issue, the efficiency of the algorithms is another crucial step. Model updating is an optimization problem to minimize the difference between the analytical responses and experimental ones. Gradientbased techniques have a wide range of applications. However, the methods may be computationally expensive and are not feasible to achieve complicated engineering problems [22]. Intelligent algorithms (GA, PSO, etc.) can be used to reduce the difference between the FEM and the measured behavior [23]. Guo et al. [24] conducted the updating procedure by the hybrid genetic/pattern-search optimization algorithm. Boulkaibet et al. [25] adopted fish school search algorithm (FSS) and PSO to update two real structures. Whale optimization algorithm (WOA), which mimics the hunting behavior of humpback whales in nature, is a swarm-based optimization algorithm introduced by Mirjalili [26]. An improved whale optimization algorithm (WOA) based on Lévy flight (LWOA) is applied to infinite systems identification and classic engineering design problems $[27,28]$.

To the authors' best knowledge, ELM and LWOA have not been explored to solve the FEMU problem. To expand their applications and improve the efficiency of FEMU, in this paper, LWOA is firstly used to optimize input weights and the biases of ELM. Then, a LWOA-ELM based surrogate model is established by the updating parameters and corresponding FRF data to replace the initial FEM. The objective function is established using the residual between the analytical values and the experimental ones. Finally, the LWOA is employed to search the best updating parameters of FEMU.

The rest of the paper is organized as follows. Section 2 presents the FRF and the objective function. The concepts of ELM, LWOA, and optimized ELM are provided in Section 3. In Section 4, an example is given to illustrate the FEMU based on the new method. The paper is concluded in Section 5 .

\section{Frequency Response Function and Objective Function}

The motion equation of structure with structural damping can be written as

$$
\mathbf{M} \ddot{x}+\mathbf{C} \dot{x}+\mathbf{K} x=\mathbf{F},
$$

where $\mathbf{M}, \mathbf{C}$, and $\mathbf{K}$ are $n \times n$ matrices of mass, damping, and stiffness, respectively; $x, \dot{x}$, and $\ddot{x}$ are $n \times 1$ vector of the structural displacement, velocity, and acceleration; and $\mathbf{F}$ is a $n \times 1$ vector of applied force.

Assuming a harmonic input, the corresponding analytical frequency response matrix is given by

$$
\mathbf{H}(\omega)=\left(-\omega^{2} \mathbf{M}+\mathrm{i} \omega \mathbf{C}+\mathbf{K}\right)^{-1},
$$

where $\omega$ is the frequency of excitation.

The acceleration FRF (AFRF) can be calculated by

$$
\mathbf{H}(\omega)=\left(\mathbf{M}-\mathrm{i} \frac{\mathbf{C}}{\omega}-\frac{\mathbf{K}}{\omega^{2}}\right)^{-1} .
$$

FEMU utilizes information from the actual structural responses to update the parameters of the FEM. Model updating problem can be formulated in the following form:

$$
\min F(x), \text { s.t } x_{\mathrm{lb}}<x_{i}<x_{\mathrm{ub}}, \quad i=1,2, \ldots, m,
$$

where $F(x)$ is the objective function; $x_{i}$ is the $i$ th updating parameter; and $x_{\mathrm{lb}}$ and $x_{\mathrm{ub}}$ are the lower and upper bound of updating parameters, respectively.

Based on the AFRF data, the research sets the residual between the FEM data and experimental ones as the objective function. It can be described as

$$
\begin{aligned}
F & =\sum_{i=1}^{n}\left|y_{t}\left(\omega_{i}\right)-y_{a}\left(\omega_{i}\right)\right|, \\
y\left(\omega_{i}\right) & =\log _{10} \mathbf{H}\left(\omega_{i}\right),
\end{aligned}
$$

where $n$ is the number of selected frequency; $\omega_{i}$ is the selected frequency points; $y_{t}\left(\omega_{i}\right)$ is the experimental response at $\omega_{i} ; y_{a}\left(\omega_{i}\right)$ indicates the corresponding response of the FEM. Considering the peak values at specified frequencies, which may overwhelm the influence of the smaller values, the logarithmic $\left(\log _{10} \mathbf{H}\left(\omega_{i}\right)\right)$ is adopted.

\section{Basic Theory}

3.1. Extreme Learning Machine. Extreme learning machine (ELM) for single-hidden layer feedforward neural networks (SLFNs) proposed by Huang has been used extensively and successfully in different fields with fast learning speed and good generalization capability. Just like the traditional feedforward network algorithm, the ELM consists of three layers of neurons: an input layer, a hidden layer, and an output layer (as shown in Figure 1). $\mathbf{w}$ is the input weight vector between the input nodes and the hidden nodes; $\beta$ is the output weight vector between the hidden nodes and the output ones.

Suppose the $N$ training dataset $\left(\mathbf{x}_{i}, \mathbf{t}_{i}\right)$, $\mathbf{x}_{i}=\left[x_{i 1}, x_{i 2}, \ldots, x_{i n}\right]^{\mathrm{T}} \in \mathbf{R}^{n}, \quad \mathbf{t}_{i}=\left[t_{i 1}, t_{i 2}, \ldots, t_{i m}\right]^{\mathrm{T}} \in \mathbf{R}^{m}$ where $\mathbf{x}_{i}$ is the input vector and $\mathbf{t}_{i}$ is the output vector. The output is given by

$$
\sum_{i=1}^{L} \beta_{i} g\left(\mathbf{w}_{i} \mathbf{x}_{j}+b_{i}\right)=\mathbf{t}_{j}, \quad j=1,2, \ldots, N .
$$

Equation (7) can be written as 


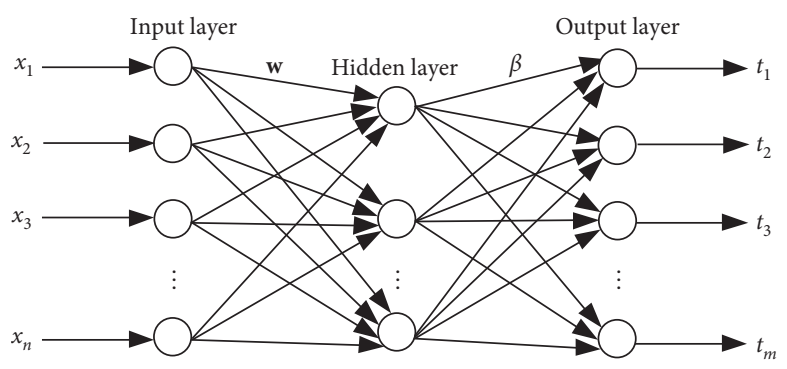

FIGURE 1: Extreme learning machine.

$$
\mathbf{H} \beta=\mathbf{T}
$$

The hidden layer output matrix $\mathbf{H}$ is given by

$$
\begin{aligned}
\mathbf{H} & =\left(\begin{array}{ccc}
\mathrm{g}\left(\mathbf{w}_{1} \mathbf{x}_{1}+b_{1}\right) & \ldots & \mathrm{g}\left(\mathbf{w}_{L} \mathbf{x}_{1}+b_{L}\right) \\
\vdots & \ddots & \vdots \\
\mathrm{g}\left(\mathbf{w}_{1} \mathbf{x}_{N}+b_{1}\right) & \cdots & \mathrm{g}\left(\mathbf{w}_{L} \mathbf{x}_{N}+b_{L}\right)
\end{array}\right)_{N \times L}, \\
\beta & =\left(\begin{array}{c}
\beta_{1}^{\mathrm{T}} \\
\vdots \\
\beta_{L}^{\mathrm{T}}
\end{array}\right)_{L \times m}, \\
\mathbf{T} & =\left(\begin{array}{c}
\mathbf{t}_{1}^{\mathrm{T}} \\
\vdots \\
\mathbf{t}_{N}^{\mathrm{T}}
\end{array}\right)_{N \times m},
\end{aligned}
$$

where $L$ denotes the number of hidden nodes; $b_{i}$ is the bias; $g(\cdot)$ is the activation function. The minimum norm least squares of equation (8) can be calculated by

$$
\widehat{\beta}=\mathbf{H}^{+} \mathbf{T}
$$

where $\mathbf{H}^{+}$is the Moore-Penrose generalized inverse of $\mathbf{H}$.

Given the training samples, active function $g(\cdot)$, and hidden nodes number $L$, the specific process of ELM can be divided into three steps:

Step 1: randomly assign input weight vector $\mathbf{w}$ and biases $b$

Step 2: calculate the hidden layer output matrix $\mathbf{H}$

Step 3: calculate the output weight $\hat{\beta}$

\subsection{Lévy Flight-Based Whale Optimization Algorithm.} Whale optimization algorithm (WOA) is a new metaheuristic algorithm, which simulates the social behavior of humpback whales. The basic WOA is divided into three operations: encircling, Bubble-net attacking behavior (exploitation phase), and search for prey (exploration period), where Bubble-net attacking consists of two approaches: shrinking encircling mechanism and spiral. Due to a lack of population diversity, WOA is easy to fall into local optimum. Lévy flight trajectory, which is the random walk step drawn from a Lévy distribution, is adapted to prevent WOA from local optimum and enhance the solution accuracy. The procedure of the LWOA is shown in Algorithm 1.
Firstly, humpback whales try to update their position towards the best search agent. The behavior is given as follows:

$$
\begin{aligned}
\mathbf{X}(t+1) & =\mathbf{X}^{*}(t)-\mathbf{A} \cdot \mathbf{D}, \\
\mathbf{D} & =\left|\mathbf{C} \cdot \mathbf{X}^{*}(t)-\mathbf{X}(t)\right|,
\end{aligned}
$$

where $\mathbf{A}=2 a \cdot \mathbf{r}-a ; a=2-2\left(t / t_{\max }\right) ; \quad \mathbf{C}=2 \cdot \mathbf{r} ; \mathbf{r}$ is a random vector in $[0,1]$; $t$ is the current iteration; $t_{\max }$ is the maximum iteration number; $\mathbf{X}(t)$ is the position vector; $\mathbf{X}^{*}(t)$ is the position vector of the best solution obtained so far.

Then, the humpback whales choose shrinking encircling mechanism or the spiral model with a probability of $50 \%$ to update the position, according to the following equation:

$$
\mathbf{X}(t+1)= \begin{cases}\mathbf{X}^{*}(t)-\mathbf{A} \cdot \mathbf{D}, & \text { if } p<0.5 \\ \mathbf{D}^{\prime} \cdot e^{b l} \cdot \cos (2 \pi l)+\mathbf{X}^{*}(t), & \text { if } p \geq 0.5\end{cases}
$$

where $\mathbf{D}^{\prime}=\left|\mathbf{X}^{*}(t)-\mathbf{X}(t)\right|$ indicates the distance between the best solution obtained so far and the current position; $b$ is a constant for defining the shape of the logarithmic spiral; $l$ is a number in $[-1,1] ; p$ is a random number in $[0,1]$.

And then, humpback whales search randomly to update the position in the exploration phase. The mathematical model is as follows:

$$
\begin{aligned}
\mathbf{X}(t+1) & =\mathbf{X}_{r}(t)-\mathbf{A} \cdot \mathbf{D}, \\
\mathbf{D} & =\left|\mathbf{C} \cdot \mathbf{X}_{r}(t)-\mathbf{X}(t)\right|,
\end{aligned}
$$

where $\mathbf{X}_{r}(t)$ is a random position vector chosen from the current population.

$|A|$ is the parameter to choose mechanism to update position. If $|\mathbf{A}| \geq 1$, a random search agent is performed by equations (15) and (16). And if $|\mathbf{A}|<1$, humpback whales update the position of the current search agent according to equations (12) and (13).

Finally, the Lévy flight trajectory is employed to balance the exploitation and exploration of WOA. The mathematical model can be formulated by

$$
\mathbf{X}(t+1)=\mathbf{X}(t)+\mu \operatorname{sign}\left[\text { rand }-\frac{1}{2}\right] \oplus \mathbf{L e v y},
$$

where Levy $\sim \mu /|v|^{1 / \beta} ; \mu$ and $v$ obey normal distribution, $\mu \sim N\left(0, \sigma_{\mu}^{2}\right) \quad$ and $\quad v \sim N\left(0, \sigma_{v}^{2}\right) ; \quad \sigma_{\mu}=[\Gamma(1+\beta) \times \sin (\pi \times$ 
$\left.\beta / 2) / \Gamma((1+\beta) / 2) \times \beta \times 2^{(\beta-1) / 2}\right]^{1 / \beta}$ and $\sigma_{v}=1 ; \oplus$ means entry-wise multiplications; $\beta$ is an index.

3.3. Optimized ELM. The prediction performance of ELM model can be assessed by root mean square error (RMSE) criterion and $R^{2}$ criterion:

$$
\begin{gathered}
\text { RMSE }=\sqrt{\frac{1}{N} \sum_{j=1}^{N}\left(y_{j}-\hat{y}_{j}\right)^{2},} \\
R^{2}=1-\frac{\sum_{j=1}^{N}\left(y_{j}-\hat{y}_{j}\right)^{2}}{\sum_{j=1}^{N}\left(y_{j}-\bar{y}\right)^{2}}
\end{gathered}
$$

where $N$ is the number of observations; $y_{j}$ is the analytical value calculated by FEM; $\hat{y}_{j}$ is the response value predicted by ELM; $\bar{y}$ indicates the average value of $y$. A value of $R^{2}$ close to 1 and RMSE close to 0 are always preferred, which reflect high accuracy of ELM.

In order to further improve accuracy of updating results, more reliable ELM models need to be created. The input weights and the biases are randomly generated in the ELM, which affect the prediction accuracy of the model. LWOA is utilized to optimize the input weights and biases of ELM. The minimized RMSE obtained by training samples is taken as the fitness function. It can be expressed as

$$
\min \mathrm{RMSE}=\sqrt{\frac{1}{N} \sum_{j=1}^{N}\left(y_{j}-\hat{y}_{j}(\mathbf{w}, b)\right)^{2} .}
$$

In the LWOA, the input weights and the biases are considered to be the humpback whales. The dimension of search space is $2 \times L+L$, where $L$ denotes the number of hidden nodes. The optimal parameters $\mathbf{w}$ and $b$ corresponding to the minimum fitness value are obtained.

\section{Model Updating Based on ELM and LWOA}

4.1. Model Updating Procedure. FEMU is an inverse problem to update the parameters by minimizing the discrepancy between the measured responses and the analytical ones from FEM. In this study, LWOA-ELM model is constructed to replace the FEM, and LWOA is also applied to minimize the objective function and obtain the results. The flowchart of model updating using LWOAELM and LWOA is shown in Figure 2. The main steps can be illustrated as follows:

Step 1: create the FEM of the structure by the ANSYS software.

Step 2: select updating parameters (modulus of elasticity and material density are selected as the updating parameters in this paper).

Step 3: construct the LWOA-ELM model by training samples, and check whether the accuracy criterion is satisfied $\left(R^{2}>0.9\right.$, RMSE $\left.<1.5 \times 10^{-4}\right)$ by the model.
Step 4: search the global minimum solution of equation (5) by LWOA, and check whether the convergence criterion (maximum iterations) is satisfied.

Step 5: obtain the model updating results.

4.2. Numerical Study. A plane structure with modulus of elasticity of the element $E=210 \mathrm{GPa}$, material density $\rho=$ $7850 \mathrm{~kg} / \mathrm{m}^{3}$ is used to test the proposed method for model updating. The initial FEM is established using ANSYS software. Figure 3 shows the FEM of the plane truss. The structure has 26 nodes and 49 finite elements. Each node (except 1 and 25) includes two translations DOFs. In this paper, nodes 24 and 19 are selected as the exciting and measuring points, respectively.

Modulus of elasticity and material density of the FEM are selected as the updating parameters. To simulate initial FEM value, increase the modulus of elasticity by $10 \%$, and decrease density by $10 \%$. The parameters are shown in Table 1.

Latin hypercube sampling (LHS) [29] is used to get the design points of updating parameters. Modulus of elasticity and material density change within $\pm 20 \%$ of the FEM values. 100 random samples are obtained for ELM. The corresponding response data are generated by equation (3). Thus, ELM model is established based on the input variables (updating parameters) and corresponding outputs (AFRF data). According to literature [17] and cross validation method, the activation function is sigmoidal function, and the number of hidden nodes is set to 80 . For verification, ELM model is compared with frequently used surrogate models, RBF model and second-order RS model [14]. RBF neural network is a three-layer feedforward network with the hidden layer containing RBF units. RS is a mathematical and statistical technique and often applied in regression analysis. The models are constructed based on the same sample points. $\mathrm{RBF}$ is conducted in MATLAB neural network toolbox. The parameter spread is set to 25 by trial and error to keep a stable and minimum value of RMSE. Each algorithm is simulated 20 independent runs in MATLAB software. The results are presented as the average of 20 runs. Comparison of ELM with RS and RBF model is shown in Figure 4. It can be seen that the prediction accuracy of ELM is better than RS and RBF in Figure 4. RMSE of ELM is 0.0012 and $R^{2}$ is 1 , RMSE of RBF is 0.0161 and $R^{2}$ is 0.9989 , and RMSE of RS is 0.0314 and $R^{2}$ is 0.9963 , which indicate that ELM model builds an accurate relationship between updating parameters and responses.

To further improve the prediction ability of ELM model, the proposed LWOA-ELM model is constructed. Moreover, LWOA-ELM is compared with GA-ELM, PSO-ELM, and WOA-ELM. GA is a widely used optimization algorithm inspired from evolutionary process. PSO is a bio-inspired stochastic algorithm derived from biological entities. In order to make a fair comparison, the population size and the maximum iteration number are set to the same data. And other parameters are the best sets after trial and error. Finally, the population size is set to 20. The maximum iteration number is set to 100 . Other parameters of these algorithms are listed in Table 2. 


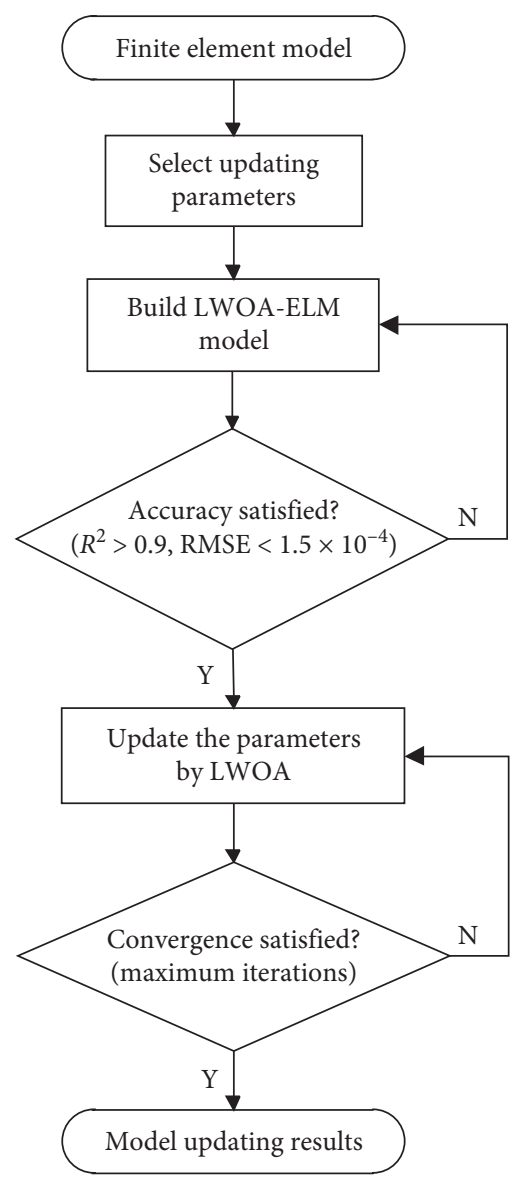

FIGURE 2: Flowchart of model updating.

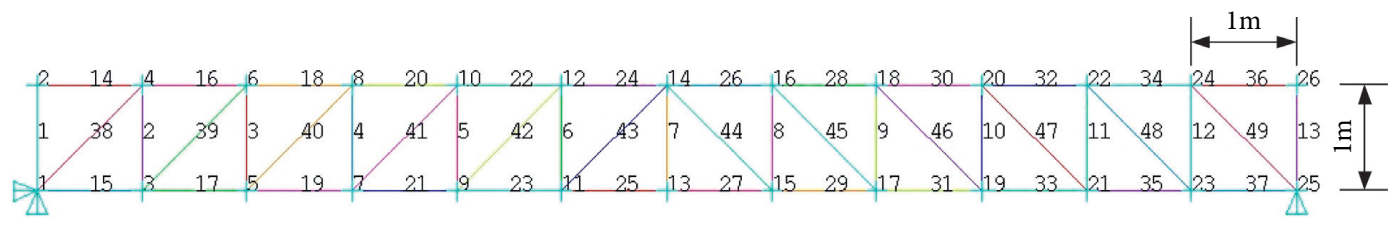

FIgURE 3: FEM of the plane truss.

The fitness convergence curves of GA-ELM, PSO-ELM, WOA-ELM, and LWOA-ELM are shown in Figure 5. From Figure 5, it is apparent that LWOA has a fast convergence than others. The smallest fitness value is LWOA. Figure 6 reflects RMSE of the four optimized ELM models.

As seen in Figure 6, the prediction accuracy of the four optimized ELM models is improved. LWOA-ELM is significantly better than GA-ELM, PSO-ELM, and WOA-ELM. The comparison results of the four algorithms are given in Table 3.

After building the LWOA-ELM model, LWOA is applied to find the best solution of the objective function of equation (5). At each time of iteration, the analytical values of FRF are predicted by LWOA-ELM instead of FEM. For the algorithm, the dimension is set as 2 (two updating parameters), and the maximum iteration number is set to 500 . The population size is set to 50 . The updated parameters are listed in Table 4. The parameters are closer to experimental parameters, which prove the validity of this method.

Actually, the experimental responses usually contain noise. $5 \%$ random noise is added to experimental FRF. The $\log _{10} H\left(\omega_{i}\right)$ before and after updating compared with the experimental data is given in Figure 7. And Figure 8 shows the FRF of initial FEM, experimental, and updated model. As shown in Figures 7 and 8, the model has a good agreement with the experimental one. The results are given in Table 5 .

From Tables 4 and 5, it can be observed that the noise affects the updating results. The maximum error is $0.86 \%$. The error is still acceptable. To further illustrate the proposed method, the first five frequencies of the FEM, experimental, and updated model are shown in Table 6.

As presented in Table 6, the relative errors decrease from $10.55 \%$ to $0.038 \%$. It is verified that the proposed method is 
Initialize relevant parameters

Generate a population $\mathbf{X}$, randomly. Calculate the fitness of each whale and get the best solution $\mathbf{X}^{*}$ while $\left(t<t_{\max }\right)$

for each search agent

Update $a, \mathbf{A}, \mathbf{C}, l$ and $p$

if $(p<0.5)$

if $(|\mathbf{A}|<1)$

Update the position of the current search agent by equation (12)

else if $(|\mathbf{A}| \geq 1)$

Select a random search agent $\mathbf{X}_{r}$

Update the position of the current search agent by equation (15) end if

else if $(p \geq 0.5)$

Update the position of the current search agent by equation (14)

end if

end for

for each search agent

Update the position of the current search agent using Lévy flight by equation (17)

end for

Check if any search agent goes beyond the search and amend it

Calculate the fitness of each whale

Update $\mathbf{X}^{*}$ if there is a better solution $\mathbf{X}^{*}$

$t=t+1$

end while

Return $\mathbf{X}^{*}$

Algorithm 1: The Lévy flight based whale optimization algorithm (LWOA).

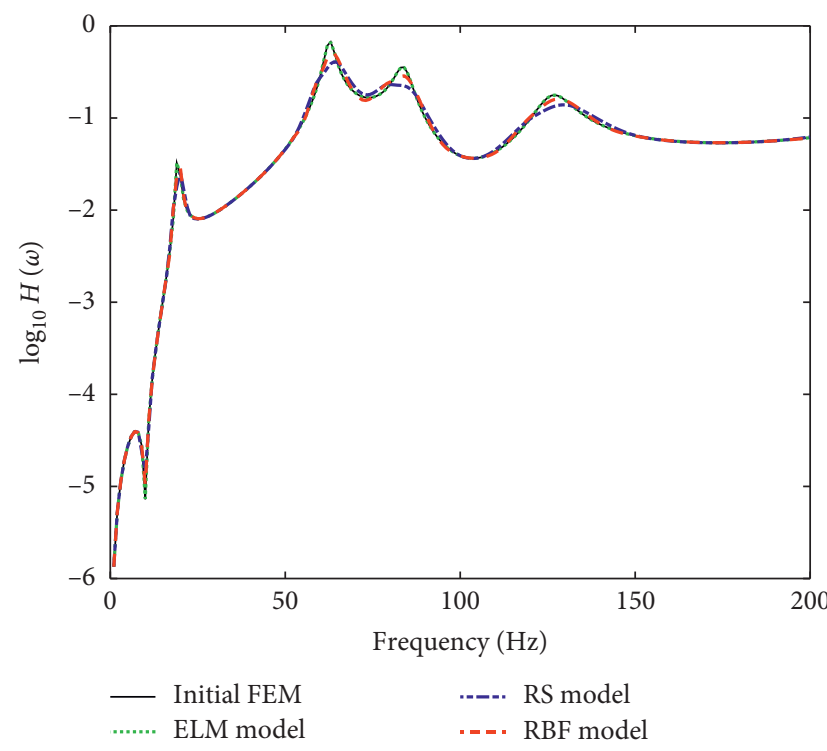

(a)

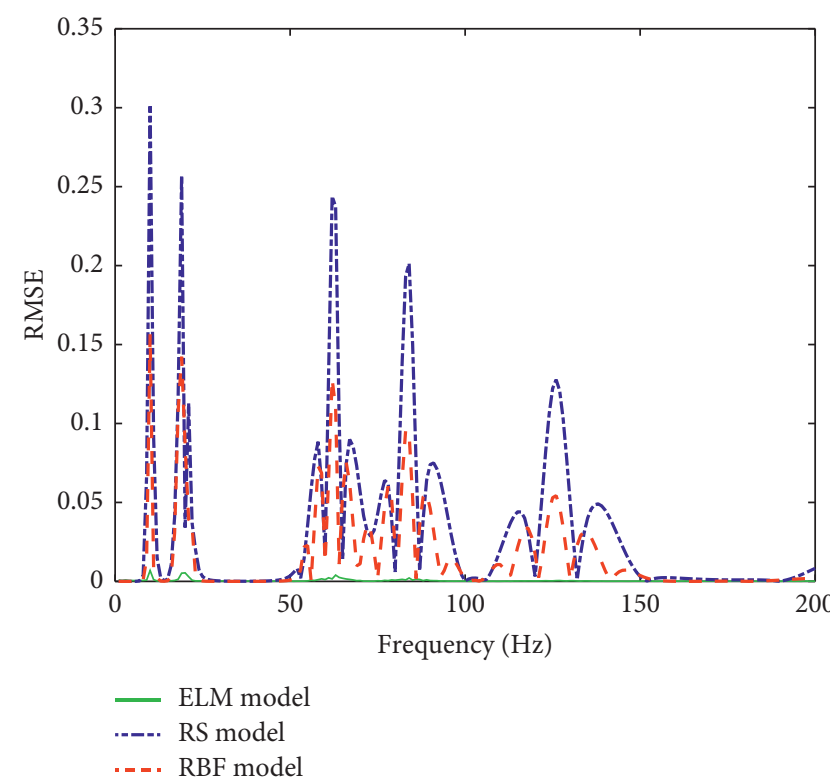

(b)

FIGURE 4: Comparison of ELM with RS and RBF model. (a) Initial FEM values and values predicted by ELM, RS, and RBF model. (b) RMSE of ELM, RS, and RBF model.

TABle 1: Parameters of experimental model and FEM.

\begin{tabular}{lcc}
\hline Updating parameters & Experimental value & Initial FEM value \\
\hline Modulus of elasticity $(\mathrm{GPa})$ & 210 & 231 \\
Material density $\left(\mathrm{kg} / \mathrm{m}^{3}\right)$ & 7850 & 7065 \\
\hline
\end{tabular}




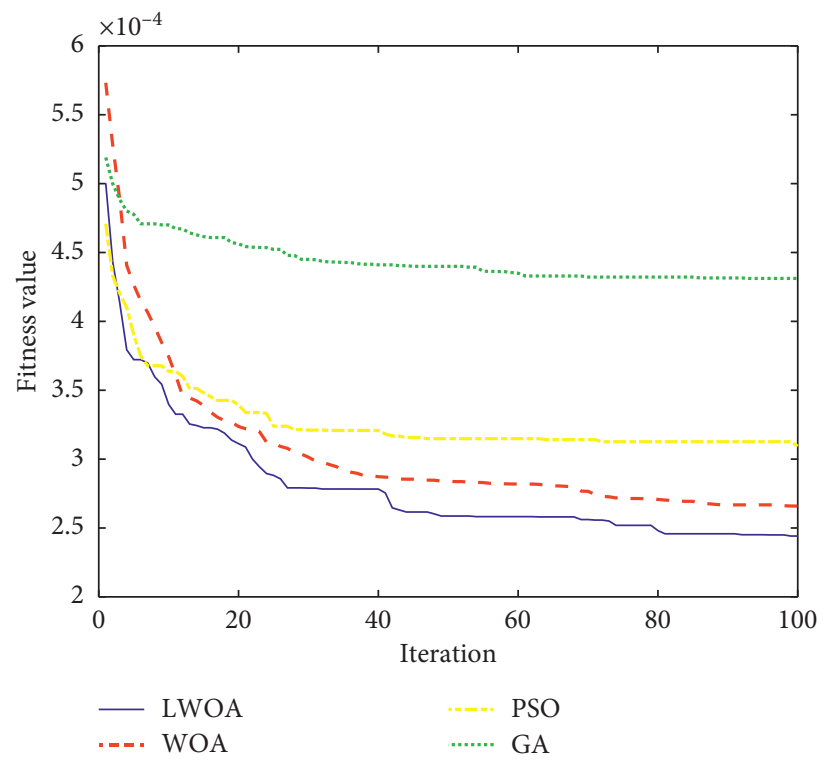

Figure 5: Convergence curves for LWOA, WOA, PSO, and GA.

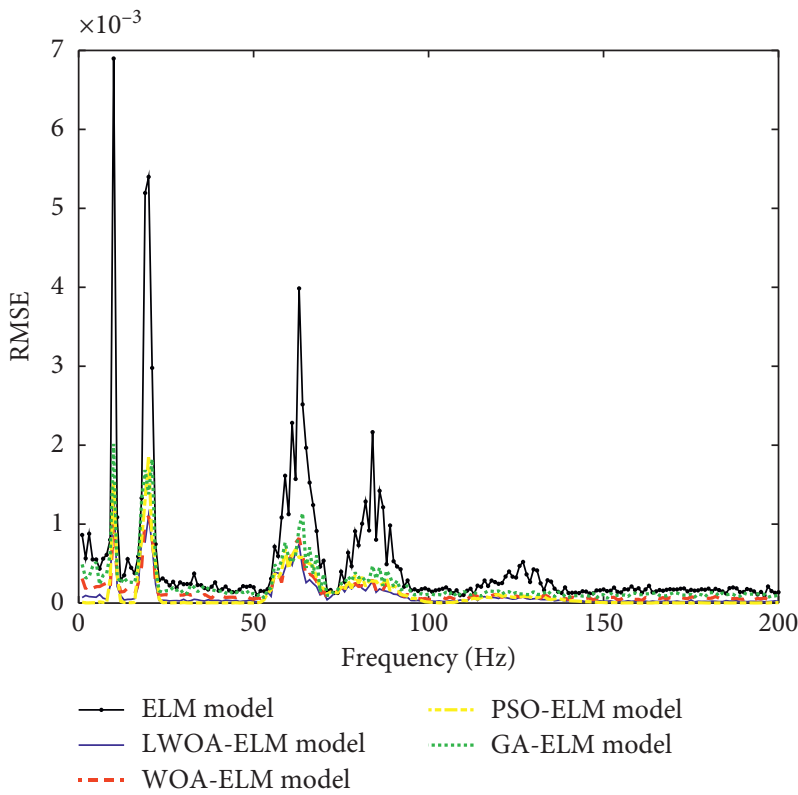

Figure 6: RMSE of the optimized ELM models.

TABLE 2: Parameters of algorithms.

\begin{tabular}{lcc}
\hline Algorithm & Parameters & Values \\
\hline \multirow{2}{*}{ GA } & Crossover probability $p_{c}$ & 0.9 \\
& Mutation probability $p_{m}$ & 0.1 \\
\hline \multirow{2}{*}{ PSO } & Learning factors $c_{1}$ & 2 \\
& Learning factors $c_{2}$ & 2 \\
\hline
\end{tabular}

TABLE 3: Comparison results of the four algorithms.

\begin{tabular}{lcc}
\hline Model & RMSE & $R^{2}$ \\
\hline GA-ELM & $2.3252 \times 10^{-4}$ & 1 \\
PSO-ELM & $1.1490 \times 10^{-4}$ & 1 \\
WOA-ELM & $1.4250 \times 10^{-4}$ & 1 \\
LWOA-ELM & $1.0263 \times 10^{-4}$ & 1 \\
\hline
\end{tabular}




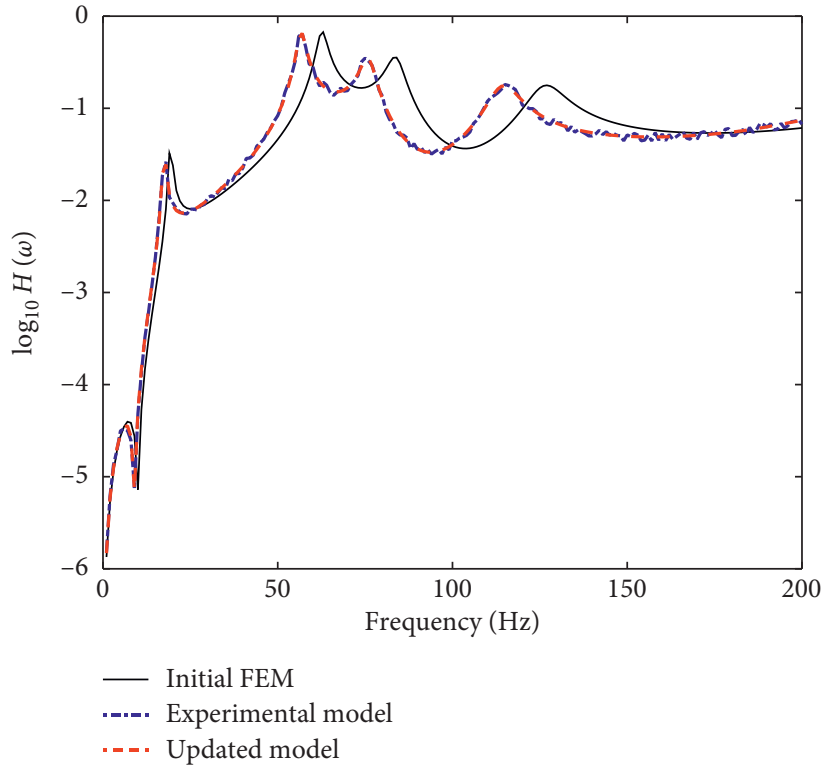

Figure 7: Result of FEMU.

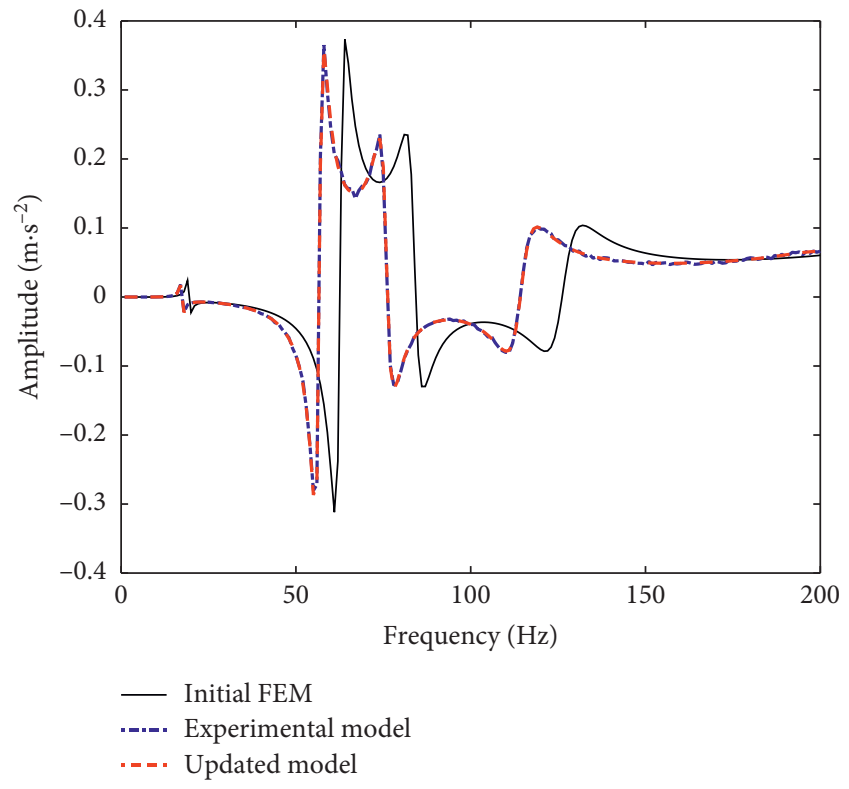

(a)

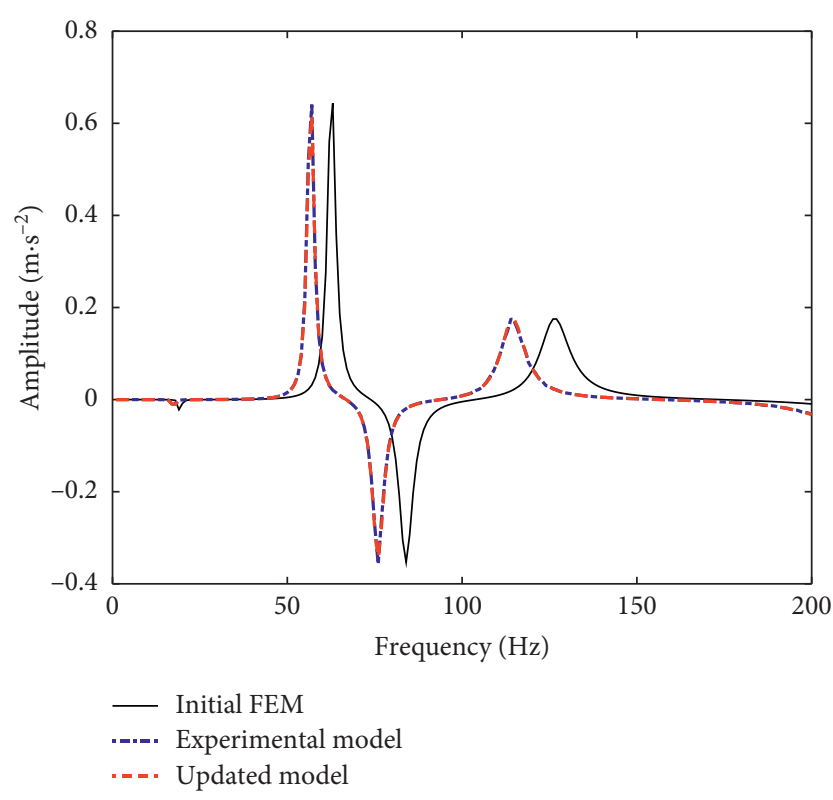

(b)

FIgURE 8: FRFs of initial FEM, experimental model, and updated model. (a) Values of the real part. (b) Values of the imaginary part.

TABle 4: Values and errors of the updated parameters without noise.

\begin{tabular}{|c|c|c|c|c|c|c|c|}
\hline Updating parameters & Experimental value & Initial value & Error (\%) & ELM & Error (\%) & LWOA-ELM & Error (\%) \\
\hline Modulus of elasticity (GPa) & 210 & 231 & 10 & 210.0131 & 0.0063 & 210.0045 & 0.0021 \\
\hline Material density $\left(\mathrm{kg} / \mathrm{m}^{3}\right)$ & 7850 & 7065 & -10 & 7851.2 & 0.0153 & 7850.4 & 0.0051 \\
\hline
\end{tabular}

TABLE 5: Values and errors of the updated parameters with 5\% noise.

\begin{tabular}{|c|c|c|c|c|c|}
\hline Updating parameters & Experimental value & Initial value & Error (\%) & LWOA-ELM & Error (\%) \\
\hline Modulus of elasticity (GPa) & 210 & 231 & 10 & 211.8 & 0.86 \\
\hline Material density $\left(\mathrm{kg} / \mathrm{m}^{3}\right)$ & 7850 & 7065 & -10 & 7911.2 & 0.78 \\
\hline
\end{tabular}


TABLE 6: Natural frequencies of the FEM, experimental, and updated model.

\begin{tabular}{lccccc}
\hline $\begin{array}{l}\text { Mode } \\
\text { order }\end{array}$ & $\begin{array}{c}\text { Initial FEM } f_{a} \\
(\mathrm{~Hz})\end{array}$ & $\begin{array}{c}\text { Experimental model } f_{t} \\
(\mathrm{~Hz})\end{array}$ & $\begin{array}{c}\text { Updated model } f_{u} \\
(\mathrm{~Hz})\end{array}$ & $\begin{array}{c}\text { Error } e_{a t}(\%) \\
\text { Error } e_{u t}(\%)\end{array}$ & $\begin{array}{c}\text { Error reduction } \\
(\%)\end{array}$ \\
\hline 1 & 19.3290 & 17.4837 & 17.4904 & & \\
2 & 62.6328 & 56.6535 & 56.6753 & & 0.038 \\
3 & 83.8904 & 75.8817 & 75.9109 & 10.55 & 99.63 \\
4 & 126.4108 & 114.3429 & 114.3869 & & \\
5 & 195.6685 & 176.9864 & 177.0545 & & \\
\hline
\end{tabular}

Note: $e_{a t}=\left|\left(f_{a}-f_{t}\right) / f_{t}\right| \times 100 ; e_{u t}=\left|\left(f_{u}-f_{t}\right) / f_{t}\right| \times 100$.

capable of updating the model, and the natural frequencies match the experimental model well.

\section{Conclusion}

A LWOA-ELM model based method for FEMU is proposed in this paper. LWOA is applied to optimize the input weights and biases of ELM. During the optimization process, LWOA-ELM model is introduced as the surrogate model to replace the initial FEM. The parameters are updated using LWOA, which obtain minimum of the objective function based on the AFRF data. The example results show that LWOA-ELM has better accuracy performance than that by the second-order RS, RBF, traditional ELM, GA-ELM, PSOELM, and WOA-ELM. With 5\% noise, the errors of updated parameters are still less than $0.9 \%$. The proposed method is suitable for FEMU. For future work, complex structure will be developed, and modal test will be performed.

\section{Data Availability}

The data used to support the findings of this study are available from the corresponding author upon request.

\section{Conflicts of Interest}

The authors declare that they have no conflicts of interest.

\section{Acknowledgments}

This research was supported by the Natural Science Foundation of China (No. 51768035) and Collaborative Innovation Team Project of Universities in Gansu Province (No. 2018C-12).

\section{References}

[1] T. Marwala, Finite-element-model Updating Using Computational Intelligence Techniques- Applications to Structural Dynamics, Springer-Verlag, London, UK, 2010.

[2] S. Sehgal and H. Kumar, "Structural dynamic model updating techniques: a state of the art review," Archives of Computational Methods in Engineering, vol. 23, no. 3, pp. 515-533, 2016.

[3] S. Sehgal and H. Kumar, "Novel dynamic model updating technique for damped mechanical system," Journal of Theoretical and Applied Mechanics, vol. 47, no. 4, pp. 75-85, 2017.

[4] S. Sehgal and H. Kumar, "Experimental damage identification by applying structural dynamic model updating," Journal of
Theoretical and Applied Mechanics, vol. 49, no. 1, pp. 51-61, 2019.

[5] V. Meruane and W. Heylen, "An hybrid real genetic algorithm to detect structural damage using modal properties," Mechanical Systems and Signal Processing, vol. 25, no. 5, pp. 1559-1573, 2011.

[6] K. Liu, R. J. Yan, and C. Guedes Soares, "An improved model updating technique based on modal data," Ocean Engineering, vol. 154, pp. 277-287, 2018.

[7] D. Hester, K. Koo, Y. Xu, J. Brownjohn, and M. Bocian, "Boundary condition focused finite element model updating for bridges," Engineering Structures, vol. 198, p. 109514, 2019.

[8] D. V. Nehete, S. V. Modak, and K. Gupta, "Coupled vibroacoustic model updating using frequency response functions," Mechanical Systems and Signal Processing, vol. 70-71, pp. 308-319, 2016.

[9] R. M. Lin and D. J. Ewins, "Model updating using FRF data," in Proceedings of ISMA, pp. 141-163, Leuven, Belgium, 1990.

[10] H. B. Başağa, T. Türker, and A. Bayraktar, "A model updating approach based on design points for unknown structural parameters," Applied Mathematical Modelling, vol. 35, no. 12, pp. 5872-5883, 2011.

[11] Y. Hong, Q. H. Pu, Y. Wang, L. J. Chen, H. Y. Gou, and X. B. Li, "Model-updating with experimental frequency response function considering general damping," Advances in Structural Engineering, vol. 21, no. 1, pp. 82-92, 2018.

[12] M. Zhan, Q. T. Guo, L. Yue, and B. Q. Zhang, "Finite element model updating of jointed structure based on modal and strain frequency response function," Journal of Mechanical Science and Technology, vol. 33, no. 10, pp. 4583-4593, 2019.

[13] T. Marwala, "Finite-element-model updating using response surface method," in Proceedings of the Structural Dynamics and Materials Conference Collection of Technical PapersAIAA/ASME/ASCE/AHS/ASC Structures, 2004.

[14] W. X. Ren and H. B. Chen, "Finite element model updating in structural dynamics by using the response surface method," Engineering Structures, vol. 32, no. 8, pp. 2455-2465, 2010.

[15] J. T. Wang, C. J. Wang, and J. P. Zhao, "Frequency response function-based model updating using Kriging model," Mechanical Systems and Signal Processing, vol. 87, pp. 218-228, 2017.

[16] Y. Zhang and Z. C. Hou, "A model updating method based on response surface models of reserved singular values," $\mathrm{Me}$ chanical Systems and Signal Processing, vol. 111, pp. 119-134, 2018.

[17] G. B. Huang, Q. Y. Zhu, and C. K. Siew, "Extreme learning machine: theory and applications," Neurocomputing, vol. 70, no. 1-3, pp. 489-5011, 2006.

[18] G. B. Huang, X. J. Ding, and H. M. Zhou, "Optimization method based extreme learning machine for classification," Neurocomputing, vol. 74, no. 1-3, pp. 155-163, 2010. 
[19] Y. M. Yang and Q. M. Jonathan Wu, "Extreme learning machine with subnetwork hidden nodes for regression and classification," IEEE Transactions on Cybernetics, vol. 46, no. 12, pp. 2885-2898, 2016.

[20] F. Han, H. F. Yao, and Q. H. Ling, "An improved evolutionary extreme learning machine based on particle swarm optimization," Neurocomputing, vol. 116, pp. 87-93, 2013.

[21] M. Eshtay, H. Faris, and N. Obeid, "Improving extreme learning machine by competitive swarm optimization and its application for medical diagnosis problems," Expert Systems with Applications, vol. 104, pp. 134-152, 2018.

[22] B. Jaishi and W. X. Ren, "Finite element model updating based on eigenvalue and strain energy residuals using multiobjective optimisation technique," Mechanical Systems and Signal Processing, vol. 21, no. 5, pp. 2295-2317, 2007.

[23] F. Shabbir and P. Omenzetter, "Particle swarm optimization with sequential niche technique for dynamic finite element model updating," Computer-Aided Civil and Infrastructure Engineering, vol. 30, no. 5, pp. 359-375, 2015.

[24] N. Guo, Z. C. Yang, L. Wang, Y. Ouyang, and X. P. Zhang, "Dynamic model updating based on strain mode shape and natural frequency using hybrid pattern search technique," Journal of Sound and Vibration, vol. 422, pp. 112-130, 2018.

[25] I. Boulkaibet, L. Mthembu, F. De Lima Neto, and T. Marwala, "Finite element model updating using fish school search and volitive particle swarm optimization," Integrated ComputerAided Engineering, vol. 22, no. 4, pp. 361-376, 2015.

[26] S. Mirjalili and A. Lewis, "The whale optimization algorithm," Advances in Engineering Software, vol. 95, pp. 51-67, 2016.

[27] Y. Ling, Y. Q. Zhou, and Q. F. Luo, "Lévy flight trajectorybased whale optimization algorithm for global optimization," IEEE Access, vol. 5, pp. 6168-6186, 2017.

[28] Y. Ling, Y. Q. Zhou, and Q. F. Luo, "Lévy flight trajectorybased whale optimization algorithm for engineering optimization," Engineering Computations, vol. 35, no. 7, pp. 2406-2428, 2018.

[29] M. D. Mckay, R. J. Beckman, and W. J. Conover, "A comparison of three methods for selecting values of input variables in the analysis of output from a computer code," Technometrics, vol. 42, no. 1, pp. 55-61, 2000. 